\title{
Augmenting Surface Interaction through Context-Sensitive Mobile Devices
}

\author{
Alex Olwal \\ School of Computer Science and Communication \\ KTH (Royal Institute of Technology), Sweden \\ alx@csc.kth.se
}

\begin{abstract}
We discuss the benefits of using a mobile device to expand and improve the interactions on a large touch-sensitive surface. The mobile device's denser arrangement of pixels and touch-sensor elements, and its rich set of mechanical on-board input controls, can be leveraged for increased expressiveness, visual feedback and more precise direct-manipulation. We also show how these devices can support unique input from multiple simultaneous users in collaborative scenarios. Handheld mobile devices and large interactive surfaces can be mutually beneficial in numerous ways, while their complementary nature allows them to preserve the behavior of the original user interface.
\end{abstract}

Keywords: Touch, mobile, interaction techniques, interactive surface.

\section{Introduction}

Touch-sensitive surfaces enable dynamic user interfaces that avoid the physical constraints of traditional input devices, and are often viewed as intuitive and easy to use. The user experience in such systems is, however, often affected by calibration issues, parallax, limited resolution, and occlusion by the user's finger. The rigid surfaces also lack the tactile feedback provided through texture, actuation, physical properties and mechanics of dedicated input controls. The interaction tends to instead rely on singleor multi-touch gestures, on-screen widgets or custom interface elements.

Several projects have investigated the use of handheld or moveable displays to address these limitations, to extend and complement the capabilities of interactive surfaces. The M-Pad system [7] augments interaction on electronic whiteboards with handheld touch-screen devices, while the Pebbles project [5] focuses on office and home appliances, and collaborative scenarios for shared displays. Spatially aware handhelds can act as tangible lenses that provide detailed information about underlying media [3, 4], increase resolution on large digital surfaces [2, 8], and improve the precision of the user's interaction [6].

This work explores how these concepts can be combined to complement a large touch-sensitive surface with a commercially available mobile touch-screen device, to leverage its denser arrangement of pixels and touch-sensor elements for precise direct-manipulation, and its rich set of mechanical on-board input controls for increased expressiveness and feedback, as illustrated in Figure 1. 


\section{Focused Mobile Interaction in the Context of a Large Display}

The content in a mobile display may be linked to the user's manipulations on an interactive surface, to enable synchronized presentations of relevant data. Such setups can leverage the respective advantages of the two devices, in which the handheld's better brightness, contrast, viewing angles and pixel density enable a high-fidelity focus, in the context of the larger surface [1]. A surface with device-tracking capabilities, such as our extended LightSense framework [6], can thus allow the mobile display to enhance the visual quality of the regions it is moved over. It can also be used as a magic lens to display alternative representations of the underlying data, such as a satellite view that is overlaid to complement a terrain map.

An alternative mode allows the user to control the view shown on the mobile display, held by the non-dominant hand, through direct pointing to the region of interest on the large surface, by the dominant hand. This mode does not depend on device tracking and enables more flexibility in posture for ergonomic viewing and interaction distances. See Figure 1.
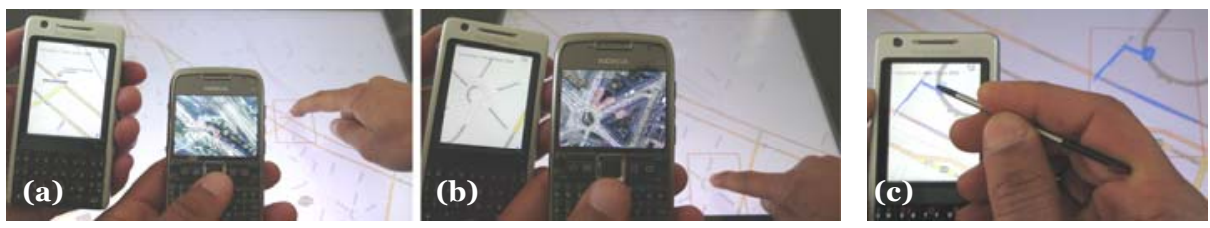

Fig. 1. a-b) Multiple mobile devices are wirelessly connected to the surface and provide individual visualizations (road maps and satellite images) of the region indicated by the user's touch. c) The higher input resolution of the mobile device's touch screen allows the user to create precise annotations, while also being able to work in a comfortable pose.

\section{Tactile Controls and Precise Touch-Screen Input}

Device movement or direct touch with the finger can also be used to indicate the point of interest for interaction, allowing the mobile device to extend the input capabilities of the surface with its tactile controls and high-resolution touch screen.

The zoom action is an example of a popular operation on interactive surfaces, and is often implemented as a "two-finger stretch" or "pinch" gesture. It does, however, rely on the surface's quality and ability to sense multiple touch points, which, for example, excludes all single-touch displays. We have, however, previously discussed how such dependencies and limitations can be avoided through complementary techniques based on interaction through a mobile touch screen or by using the controls of a tracked mobile device [6]. This work introduces an additional technique based on a jog wheel (a rotary encoder that is a side-mounted equivalent of the mouse wheel, which is conveniently located under the user's thumb on many mobile devices), to support rapid changes in zoom level about the indicated point of interest on the large display. The wheel can be rolled downwards/upwards for zooming in/out and enables fine adjustments and large changes in the same mode, due to its mechanical properties. The wheel on the Sony Ericsson P1i device used in this work moves in 24-degree 

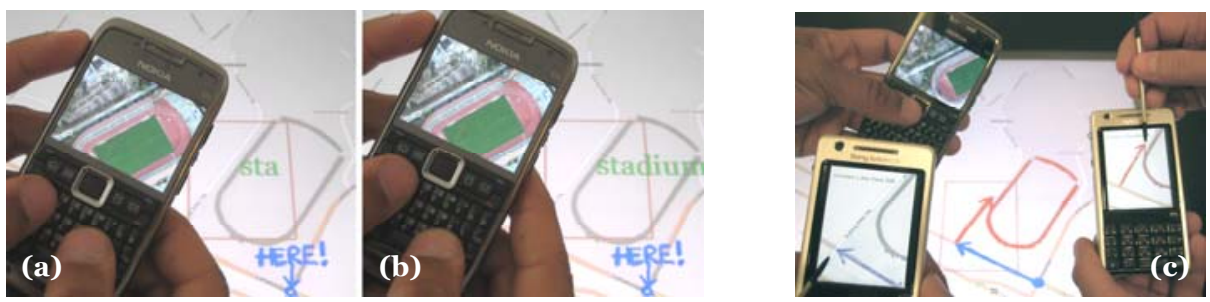

Fig. 2. a-b) The lack of tactile buttons on the surface can be addressed by using the physical keyboard of the mobile device. Here, the user enters "Stadium" on a point of interest. c) Simultaneous input from multiple uniquely identified devices allows collaborative scenarios. A blue arrow has been drawn on the left device, while a red arrow and an outline of the stadium has been added using the right device.

steps, where passive tactile feedback is provided through small "snaps" that are felt during slow rolling actions. The continuous wheel also allows very fast rolling for large zooming magnitudes, but there is, in contrast to many other approaches, little risk of losing context, as the finger is always pointing to the target location. The zooming action is also easily reversible by rolling the wheel in the opposite direction. Devices that lack jog wheels can use the up/down keys, or one of the previously described touch-screen techniques [6] for zooming.

Tactile controls are perhaps even more important for text input. A physical keyboard's form factor and need for regular maintenance often make it impractical on interactive surfaces, which instead tend to favor on-screen keyboards. The lack of tactile feedback, however, demands high visual attention, and affects typing speed. Figure 2a-b shows how a mobile device's keyboard can be used as an alternative means of text input in such systems. It shares the mechanical and tactile benefits of ordinary computer keyboards, while emphasizing portability and compactness.

Similarly to previous work $[4,5,6,7,8]$ of interaction through a mobile device's touch-screen, we provide precise annotation that is propagated to the large display, taking advantage of the higher pixel density and touch-sensor resolution of the mobile device. (See Figures 1c and 2c.)

\subsection{Input from Multiple Uniquely Identified Users}

One of the main benefits of multi-touch surfaces is the ability to support simultaneous interaction for multiple users. It is, however, not straightforward to identify an individual's touch input without the use of custom hardware and tethering. A user's mobile device can, on the other hand, provide implicit identification to allow unique input from multiple wirelessly connected users [5]. It can also surpass physical, computational or sensing constraints that affect how many touch-points single- or multitouch surfaces can detect and track.

Our implementation supports collaborative annotations and text input in individual colors from multiple mobile devices, as shown in Figure 2. A user can touch a location on the large surface to indicate the starting point for text input, and can continue typing, even if another user moves the input focus to another part of the screen. Freehand annotations from different touch-screen devices are mapped to the current rectangular focus region on the surface and visualized with unique colors. 


\subsection{Implementation}

The system uses our extended LightSense framework [6], where Java ME mobile clients communicate over Bluetooth with a Java server on the PC. The main application interfaces the touch-overlay, communicates with the Java Server over UDP and renders the graphics on the large surface using C++/OpenGL. Sony Ericsson provided P1i devices (touch screen and QWERTY-keyboard), while Nokia provided a 5800 XpressMusic (touch screen) and E71 (QWERTY-keyboard) device.

\section{Conclusions and Future Work}

This paper discusses the benefits of complementing an interactive surface with mobile devices, where bidirectional communication synchronizes graphics and user input. Such symbiotic scenarios, where the input and output mechanisms are shared over multiple connected devices, make it possible to leverage the respective advantages of mobile devices and large interactive surfaces. We provide examples of how the handheld device's higher pixel density, input resolution and rich set of onboard tactile controls, can expand the expressiveness of the large digital surfaces that provide the context for interaction. The possible input can be expanded further, by leveraging other built-in sensors in mobile devices, which could make typically exotic technology readily available on digital surfaces. Preliminary versions of the described techniques were used by trained, non-technical staff to demonstrate zooming and annotation on interactive surfaces to a large number of visitors during a five-day trade show. The staff's enthusiasm and interest in the techniques, combined with how fast they learned to use them, was encouraging. We plan to further study our techniques in formal user evaluations, with regard to usability, preference and performance.

\section{References}

1. Baudisch, P., Good, N., Bellotti, V., Schraedley, P.: Keeping things in context: A comparative evaluation of focus plus context screens, overviews, and zooming. In: Proc. CHI 2002, pp. 259-266 (2002)

2. Benko, H., Ishak, E.W., Feiner, S.: Collaborative mixed reality visualization of an archaeological excavation. In: Proc. ISMAR 2004, pp. 132-140 (2004)

3. Fitzmaurice, G.W.: Situated information spaces and spatially aware palmtop computers. Commun. ACM 36(7), 39-49 (1993)

4. Mackay, W.E., Pothier, G., Letondal, C., Bøegh, K., Sørensen, H.E.: The missing link: augmenting biology laboratory notebooks. In: Proc. UIST 2002, pp. 41-50 (2002)

5. Myers, B.A., Nichols, J., Wobbrock, J.O., Miller, R.C.: Taking Handheld Devices to the Next Level. IEEE Computer 37(12), 36-43 (2004)

6. Olwal, A., Feiner, S.: Spatially aware handhelds for high-precision tangible interaction with large displays. In: Proc. TEI 2009, pp. 181-188 (2009)

7. Rekimoto, J.: A multiple device approach for supporting whiteboard-based interactions. In: Proc. CHI 1998, pp. 344-351 (1998)

8. Sanneblad, J., Holmquist, L.E.: Ubiquitous graphics: combining hand-held and wall-size displays to interact with large images. In: Proc. AVI 2006, pp. 373-377 (2006) 